\title{
EXPERIMENTAL INVESTIGATIONS AND NUMERICAL MODELLING OF THE MELTING PROCESS IN THE COLD CRUCIBLE
}

\author{
A. Umbrashko ${ }^{(1)}$, E. Baake ${ }^{(1)}$, B. Nacke ${ }^{(1)}$ and A. Jakovics ${ }^{(2)}$ \\ ${ }^{(1)}$ Institute for Electrothermal Processes, University of Hanover, Wilhelm-Busch-Str.4, \\ D-30167 Hanover, Germany \\ (2) Laboratory for Mathematical Modelling of Environmental and Technological Processes, \\ University of Latvia, Zellu str.8, LV-1002 Riga, Latvia
}

\begin{abstract}
Temperature and velocity measurements were performed in the Aluminium melting process in the cold crucible induction furnace. The results of those experimental investigations are compared with the steady-state $2 \mathrm{D}$ calculations based on RNG k- $\varepsilon$ model, as well as with transient 3D LES modelling predictions.
\end{abstract}

\section{INTRODUCTION}

The induction furnace with cold crucible (IFCC) offers various technological and economical advantages, like high-purity cast products as well as melting, alloying and casting in one process-step [1]. But the knowledge and under-standing of the complex physical behaviour of the IFCC is required to realize an efficient and reliable melting and casting process. Practical experiences show that the overheating temperature of the entire melt, which is determined by the electromagnetic, hydrodynamic and thermal behaviour of the cold crucible installation is one of the key parameters of this technological process [2].

For the experimental investigations of the temperature and melt flow velocity field of the IFCC Aluminium serves as a model melt. Suitable measuring methods are selected, improved and realised, particularly with regard to the high melt temperature and very aggressive behaviour the Aluminium melt. The measured data is used to examine time averaged but also instationary processes in order to describe the turbulent characteristic of the melt flow. Examining the nonlinearity of the system, which strongly influenced the reachable overheating temperature of the melt, special attention is paid to the investigation of the meniscus shape of the melt and the skull formation at the bottom and at the wall of the cold crucible.

Former experimental investigations in a crucible induction furnaces have carried out that low-frequency and turbulent oscillations of the recirculating toroidal main flow eddies play an decisive role in the heat and mass exchange processes in the melt $[3,5]$. Two- and three-dimensional

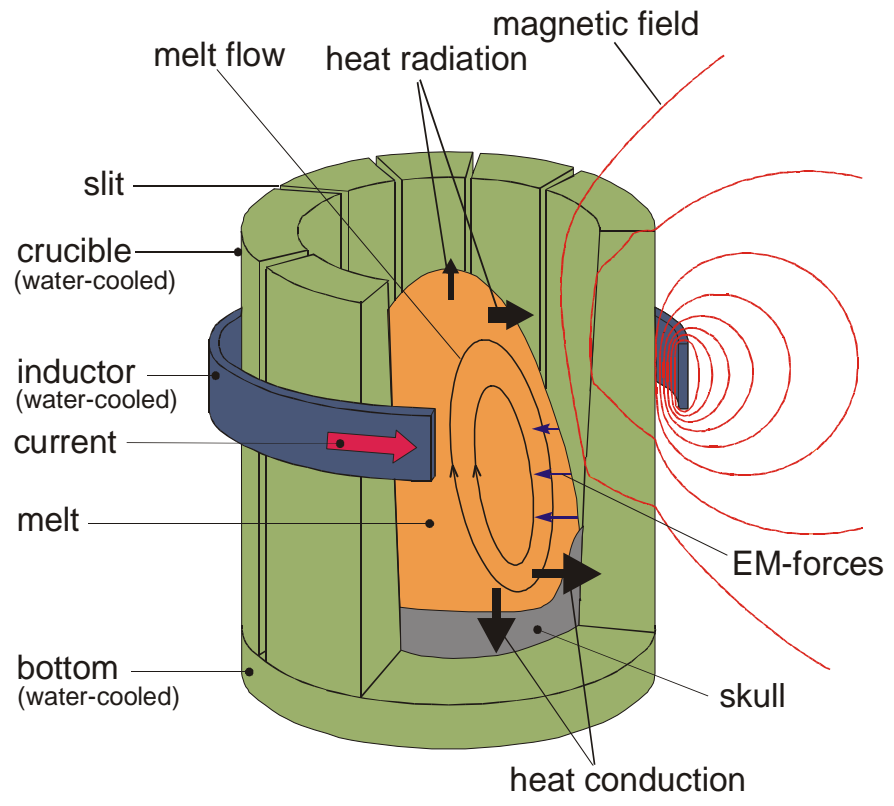

Figure 1. Schematic sketch of an IFCC. 
hydrodynamic calculations of the melt flow, using two-equation turbulence models based on Reynolds Averaged Navier-Stokes approach, do not predict such flow behaviour. That's why the Large Eddy Simulation (LES) numerical technique was approved to be an alternative for the various $\mathrm{k}-\varepsilon$ model modifications. The results of the transient 3D LES simulation revealed the large scale periodic flow instabilities, which are in good agreement with the expectations based on the data from the experiments [3]. Due to this positive results, transient 3D calculations using the LES model have been carried out in order to simulate the turbulent melt flow and temperature distribution in the cold crucible. The commercial CFD software package FLUENT is used for the application of LES to the hydrodynamic and thermal problem, but the external electromagnetic forces and the shape of the melt surface is calculated using ANSYS and self-developed FEM codes.

\section{EXPERIMENTAL SETUP}

\section{Temperature measurements}

The experimental investigations, presented in this paper, included two separate measurement's series:

1) temperature measurements;

2) velocity measurements.

Both of them were performed using $6 \mathrm{~kg}$ pure aluminium $(99.5 \%)$ in the cold crucible with a radius of $7.8 \mathrm{~cm}$ and a height of $26 \mathrm{~cm}$ (Fig.2). The output power of the generator was 200 $\mathrm{kW}$ at the frequencies $9-10 \mathrm{kHz}$. The meniscus height reached up to $\sim 22.5 \mathrm{~cm}$ under those conditions. With these process parameters the meniscus shape of the melt surface is quite stable and therefore it is possible to perform detailed investigations of the free melt surface itself, the temperature field and the turbulent melt flow.

The temperature distribution was measured using $\mathrm{NiCr}-\mathrm{Ni}$ thermocouples, which were placed in a protective ceramic tube to avoid their destruction in the very aggressive aluminium environment during long-lasting experiment. However, due to this protection, the thermal inertia of the thermocouple was quite long $(\sim 2.75 \mathrm{~s})$, therefore, it was possible to measure only time-averaged temperature values. In order to investigate temperature oscillations in several characteristic points of the melt, the thermocouple was used without ceramic protection. In this case the response time became approximately $0.8 \mathrm{~s}$, but the operational time for one thermocouple decreased to the 10-15 minutes. Obtained temperature oscillations (Fig.3) can be compared with the results of the transient 3D modeling using Large

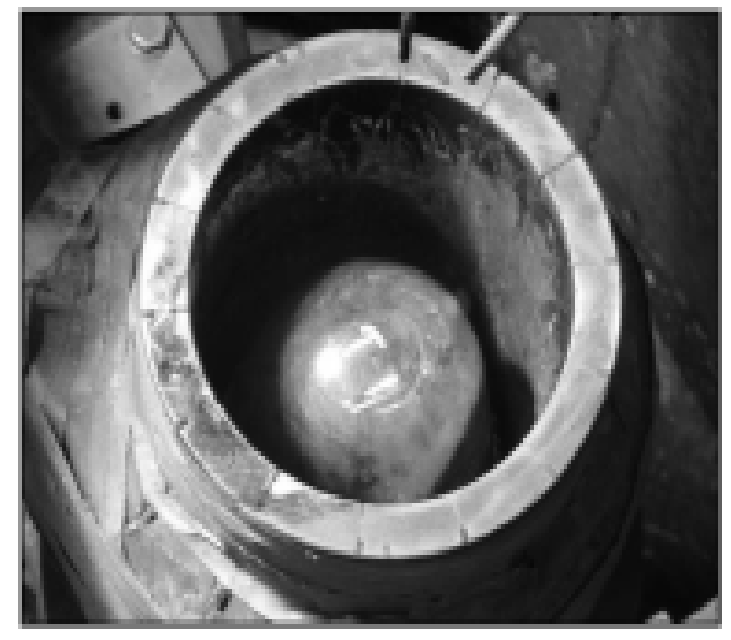

Figure 2. Cold crucible installation with Aluminium melt

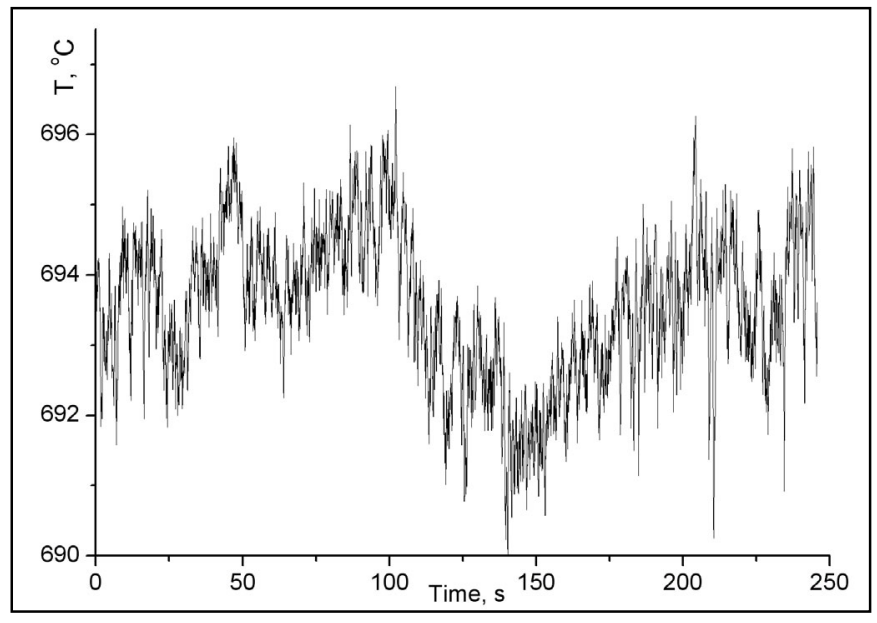

Figure 3: Measured temperature oscillations in melt of the cold crucible (at $\mathrm{h}=9 \mathrm{~cm}, \mathrm{r}=3 \mathrm{~cm}$ ) 
Eddy Simulation (LES). The very long-period temperature changes, which are visible on that graph, can be explained with unstable thermal regime of the entire melt, e.g. when some part of the skull become melted and mixed with other material. And, if we apply the high-pass filter to those data, then oscillations amplitude is within range of 1-2 degrees.

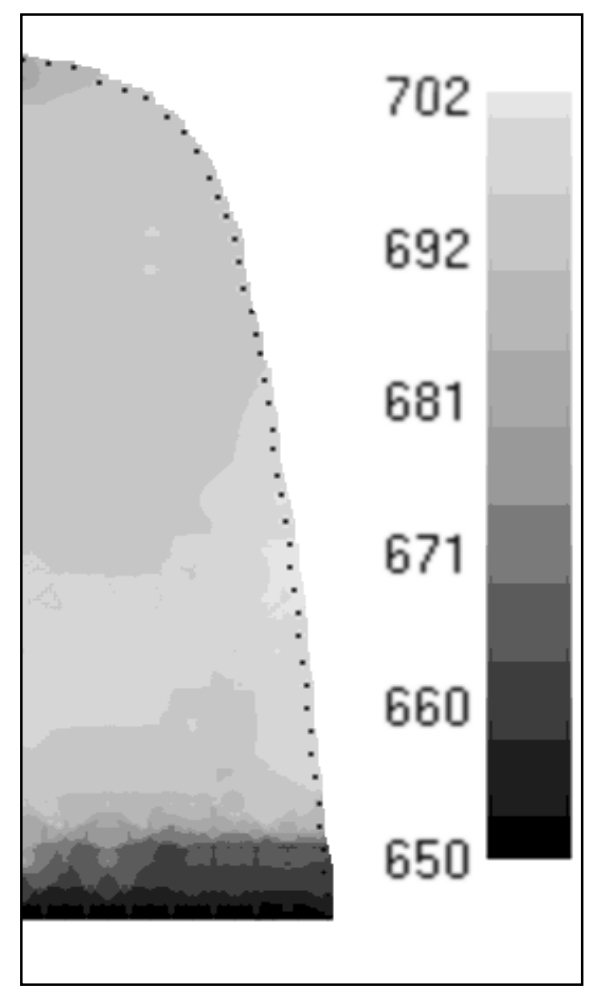

Figure 4. Measured temperature distribution $\left[{ }^{\circ} \mathrm{C}\right]$

The time-averaged temperature field as it was measured is shown on the Fig.4. There is clearly seen how temperature distribution is influenced by the thermal boundary conditions. The lowest temperatures are at the water-cooled bottom, where was detected the solid skull layer with thickness about $10 \mathrm{~mm}$. Also the radiation losses from the free surface lead to the formation of relatively cold area at the top. And the highest temperatures are observed in the intensive inductive heating region. The temperature distribution in the rest of the melt is more or less homogeneous.

\section{Velocity measurements}

The melt flow velocity was measured with electromagnetic sensor, which's principle of operation is based on the electromagnetic law of Faraday

$$
\vec{E}=-(\vec{V} \times \vec{B}) .
$$

The metal melt works as moving with velocity $\vec{V}$ conductor, which flows perpendicular across magnetic field $\vec{B}$ of the magnet. At that the electric field is created directed perpendicular to both vectors. This field is proportional to the velocity of the flowing metal and is measured as the voltage across the pair of electrodes.

For complete defining of the three-dimensional turbulent distribution of flow all three spatial components of velocity should be determined. Because it is impossible to measure all three components simultaneously, first, using $90^{\circ}$ chamfered holder of the probe rod the radial and axial components of the flow velocity are measured, then with the help of the direct holder - radial and azimuth ones.

With the melt temperature higher than the maximum working temperature of the sensors with the permanent magnet (about $450^{\circ} \mathrm{C}$ ) there appears distinct, partially irreversible, not reproducible weakening of magnetization. That is why the sensors with the permanent

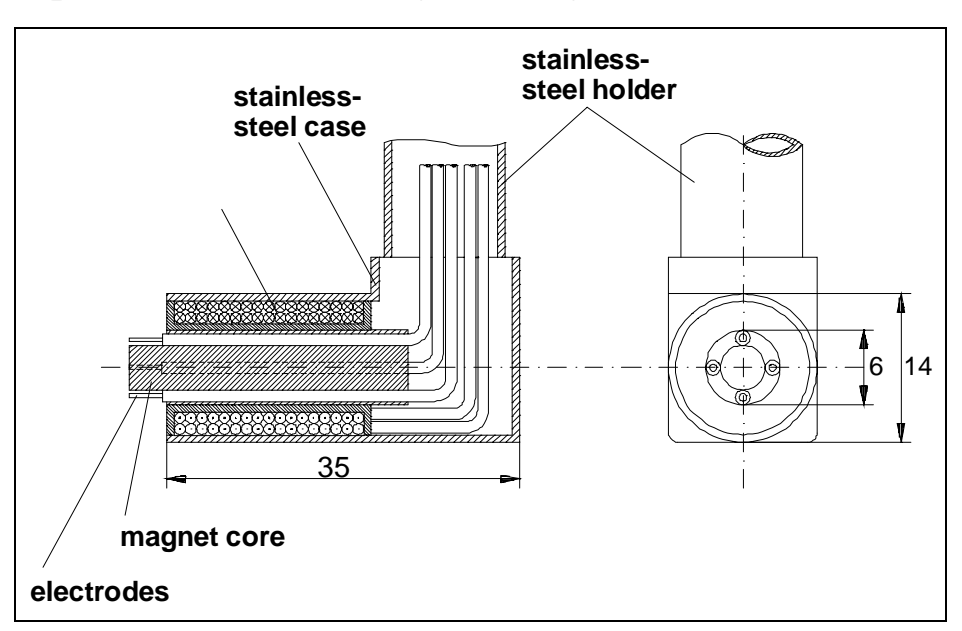

Figure 5. The structure of the electromagnetic sensor [2] magnet, measuring the flux, have very limited application in liquid aluminium. No induction methods for measuring local flow velocity in liquid metals within the temperature range between $600^{\circ} \mathrm{C}$ and $700^{\circ} \mathrm{C}$ are known from the literature [4]. That is why for the application in the aluminium melt the electromagnetic velocity sensor shown in Fig. 4 was developed and made [5].

Unlike the probe rod with the permanent magnet, the magnetic field of the electromagnetic 
sensor is created by the excitation coil along which the direct current is flowing. The case of the sensor, magnetic core and the coil case are made of steel. During the measurements the coil current was kept on the level of 10 A. The indicated level of the current was chosen taking into consideration the provision possibly high strength of the field with the limited density of current in the coil wire.

Low corrosion durability of the flow velocity measuring sensor in the aluminium melt appeared to be the greatest problem in the measurements performed. After 10-20 minutes of operation the magnetic steel core and the steel case were so greatly corroded that the sensor became unfit for the further operation. To prolong the lifetime of the sensor at the expense of other materials application such as platinum or tungsten, is impossible as they do not have long durability against the aluminium melt [4]. The attempts to prolong the operation period of the sensor protecting it with the ceramic cover did not bring the desired results as well.

The device for measuring velocity of aluminium alloys consists of electromagnetic sensor, source of direct current of the sensor coil, system of the sensor fixation in the specified point of the melt, data acquisition system and connecting wires and cables.

All sensors were calibrated in the induction crucible furnace with Wood's metal, which has melting temperature of $72{ }^{\circ} \mathrm{C}$. The measured sensitivity of the sensors varied through the range $0.3-0.8 \mu \mathrm{V} /\left(\mathrm{cm}^{*} \mathrm{~s}^{-1}\right)$.

The data acquisition system is based on Top Message device from Delphin firm and portable computer Pentium III $700 \mathrm{MHz}$, connected into network TCP/IP Ethernet. The Top Message device has a 24 bit A/D converter and provides the processing of signals from 15 analogue inputs. The high precision of the converter allowed to refuse of using DC preamplifiers and connected with this zero drift and additional disturbances. Finally to solve the problem of zero drift appeared to be possible at the expense of periodical changing of the direct

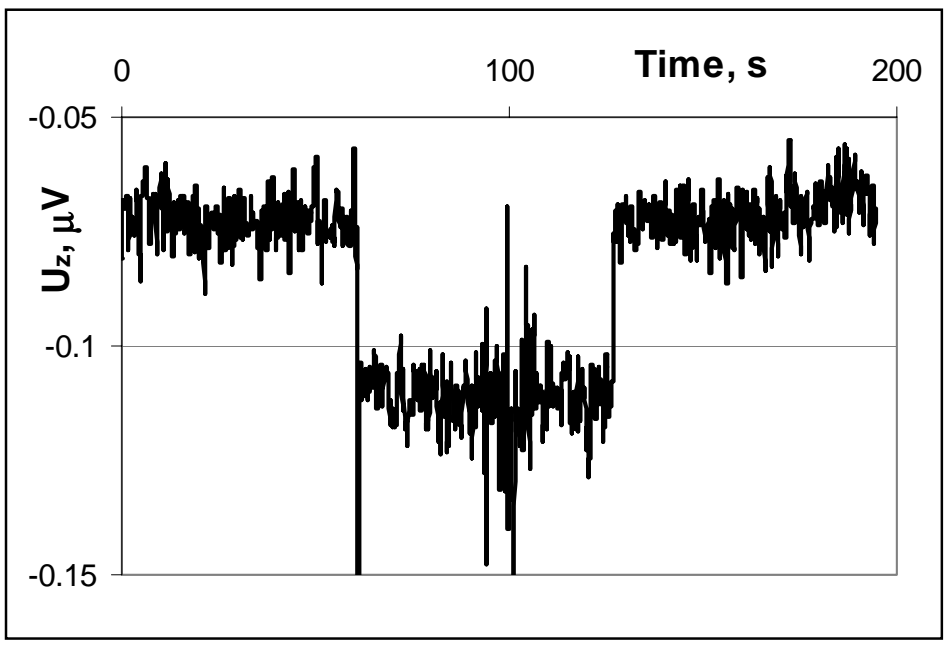

Figure 6. Typical signal from axial velocity sensor (with two polarity changes). current power supply source polarity in the process of measurement (Fig.6).

The measuring system possibilities allowed to refuse of using physical filters for suppression the alternating current disturbances and to change them for mathematical filtration of the signal. In the process of preparatory work the best result was attained while averaging the signal from the sensor during 10 seconds.

The main results of our velocity measurements in the liquid aluminium show, that flow pattern consists of two vortexes and the zone of their interaction is located between $\mathrm{z}=7$ and $\mathrm{z}=9 \mathrm{~cm}$. The maximum axial velocity detected in the upper vortex on the symmetry axis was $40 \pm 5 \mathrm{~cm} / \mathrm{s}$. In overall, these observations are in quite good agreement with numerical predictions, as it will be described later in the next chapter.

\section{NUMERICAL MODELLING}

\section{D steady-state modelling}

We have chosen the RNG modification of the $\mathrm{k}-\varepsilon$ model for the $2 \mathrm{D}$ simulation of the KIT process. According to our previous numerical studies, it has delivered more accurate 
predictions about turbulence properties in the recirculated flows than the standard model. But, both of them usually underestimated the heat transfer intensity between the main flow eddies formed by the external electromagnetic forces [6]. As it is shown on the velocity plot (Fig. 7) the time-averaged flow pattern consist of two vortexes. The thermal boundary conditions for upper and lower vortexes significantly differ - we have the radiation from the free surface above and water-cooled bottom below. The estimated heat flux distribution shows that only $6 \%$ of the thermal energy are lost due to the radiation. The rest of the heat is carried away with the cooling water through the crucible bottom. As far as the heat exchange between the two parts was underestimated, the 2D steady-state simulation predicted too high temperature in the upper region of the melt, which is not confirmed by experimental data.

3D transient LES simulation

3D calculations were based on Large Eddy Simulation (LES) turbulence modelling method, which can be described as a compromise between the solving of RANS equations and Direct Numerical Simulation (DNS). Main flow structure is resolved directly like in the DNS approach, but small eddies, which size is comparable

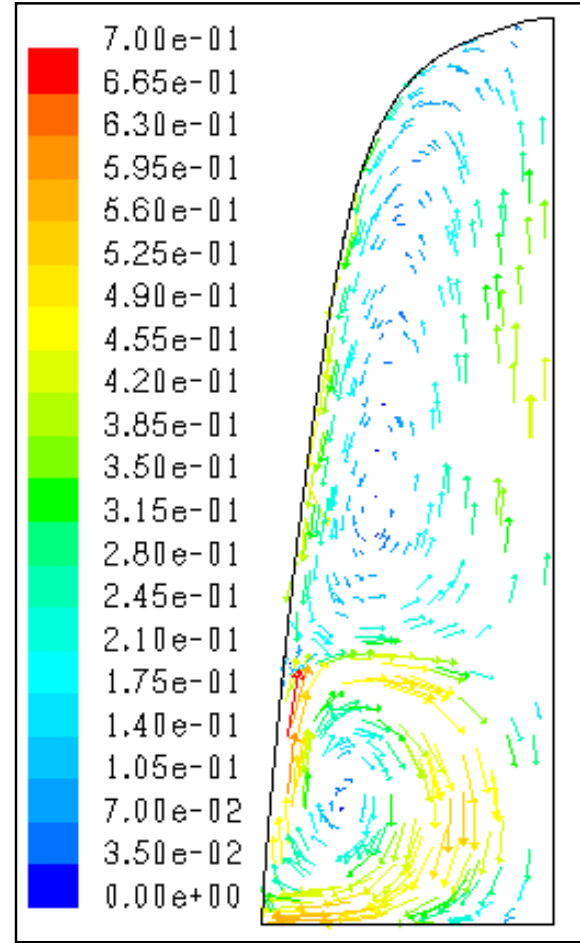

Figure 7. Velocity vectors calculated with 2D RNG k- $\varepsilon$ turbulence model $[\mathrm{m} / \mathrm{s}]$ with grid size, are modelled additionally. Therefore, finer meshing and, as result, more computational resources are required in order to get an advantage over two-equation models, e.g. k- $\varepsilon$ turbulence model, but still less than it is necessary for the application of the DNS. The subgrid turbulent viscosity $\mu_{\text {sub }}$ was calculated with Smagorinsky-Lilly scheme used in FLUENT [7-9]:

$$
\mu_{s u b}=\rho L_{s}^{2}\left|S_{i j}\right|, L_{s}=\min \left(k d, C_{s} \sqrt[3]{V_{c}}\right), S_{i j} \equiv \frac{1}{2}\left(\frac{\partial u_{i}}{\partial x_{j}}+\frac{\partial u_{j}}{\partial x_{i}}\right)
$$

where $d$ is the distance from the closest wall, $k=0.42, \mathrm{C}_{\mathrm{s}}=0.1$ is Smagorinsky constant and $V_{c}$ is volume of the computational cell.

The calculations ran on the three-dimensional model, which consisted of approx. 4 millions finite elements, with time-step $10 \mathrm{~ms}$. It took about one month for simulation of 30 seconds flow development using parallel computation with 8 processors. The resulting timeaverage velocity field (Fig. 8) looks very similar to the one predicted with 2D steady-state calculations, as well as quite good agrees with experimental observations. However, 3D transient approach allows to model accurately the heat transfer processes in such flows, where two or more recirculated eddies are interacting. The calculated flow pattern at the each timestep is not symmetrical (Fig. 10), and simulation shows, that melt intensively oscillates. Those oscillations provide convective heat transfer mechanism, which is possible to simulate numerically only using transient three-dimensional calculation techniques. The figure 9 shows the time-averaged temperature distribution calculated with LES: it is more homogeneous, than in case of 2D modelling and resembles the measured temperature field (Fig. 4). In the pictures series with temperature filed at the consequent time-steps it can be observed how relatively 


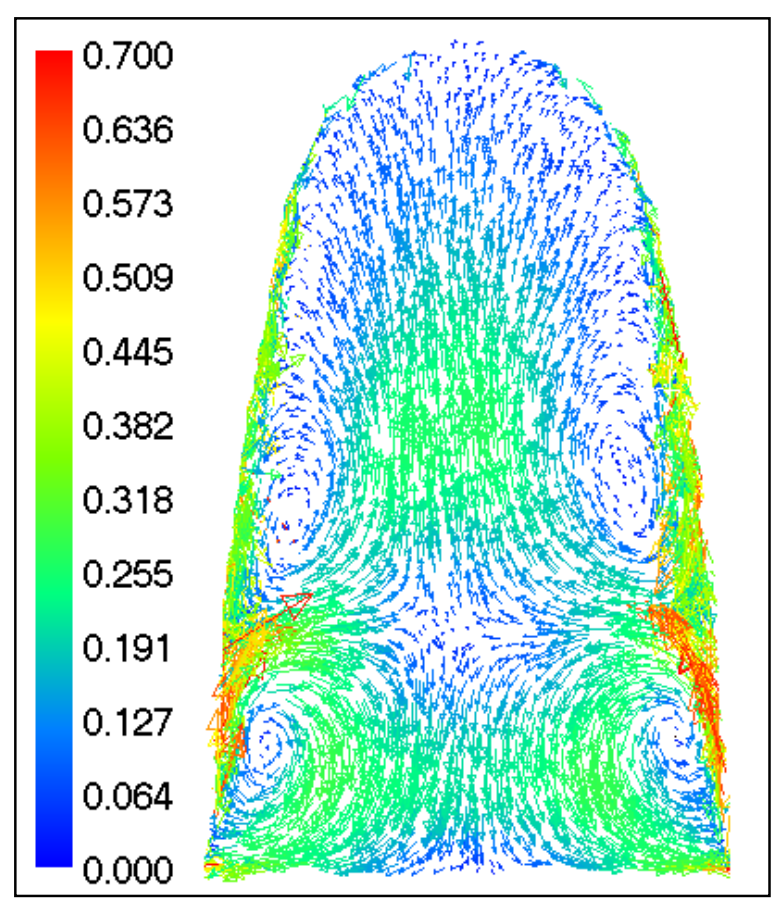

Figure 8. Time-averaged velocity field calculated with 3D transient LES turbulence model $[\mathrm{m} / \mathrm{s}]$

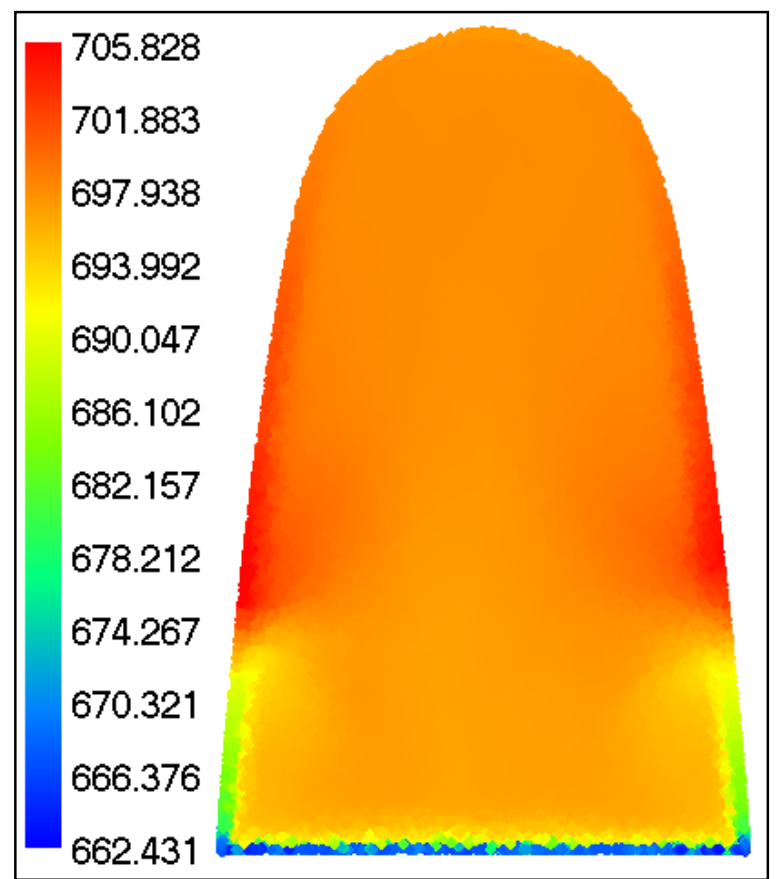

Figure 9. Time-averaged temperature field calculated with 3D transient LES turbulence model $\left[{ }^{\circ} \mathrm{C}\right]$.

cold melt masses from below penetrate into upper vortex area and are dissolved there (Fig.11).

The main noticeable disagreement with measurements is seen in the lower vortex region directly at the melt surface. The temperatures in this area seem to be underestimated in simulation, if we are comparing with the experiment, and probably this is caused by too rough boundary meshing, which leads to the overestimation of radiation heat losses form the surface. Our future research plans include investigations on the subject how the boundary mesh refinement influences the results of the calculations.

\section{Acknowledgement}

This work was carried out with the IBM pSeries Supercomputer of the HLRN and the authors thank all members from the HLRN for their support.

\section{CONCLUSIONS}

Measurements of the temperature and velocity fields in the aluminium melting process in the cold crucible show typical recirculating flow structure with an axial symmetry and with presence of intensive three-dimensional flow field oscillations, which are responsible for effective mixing and temperature homogenisation of the entire melt. The modelling results show, that only the 3D transient LES is able to model correctly these heat and mass transfer processes. Therefore, once again, it has proved to be a reliable tool for modeling of complex turbulent flows in induction furnaces.

\section{REFERENCES:}

[1] Vogt, M.; Bernier, F.; Mühlbauer, A.; Blum, M.; Jarczyk, G.: Experimental Investigations of Temperature Field and Energy Flows in Induction Furnace with Cold Crucible and a practical Application of the Results. Proc. of Int. Symp. Electromagnetic Processing of Materials, Nagoya 2000, 289-294.

[2] Baake, E.; Nacke, B.; Bernier, F.; Vogt, M.; Mühlbauer, A.; Blum, M.: Experimental and numerical investigations of the temperature field and melt flow in the induction furnace 


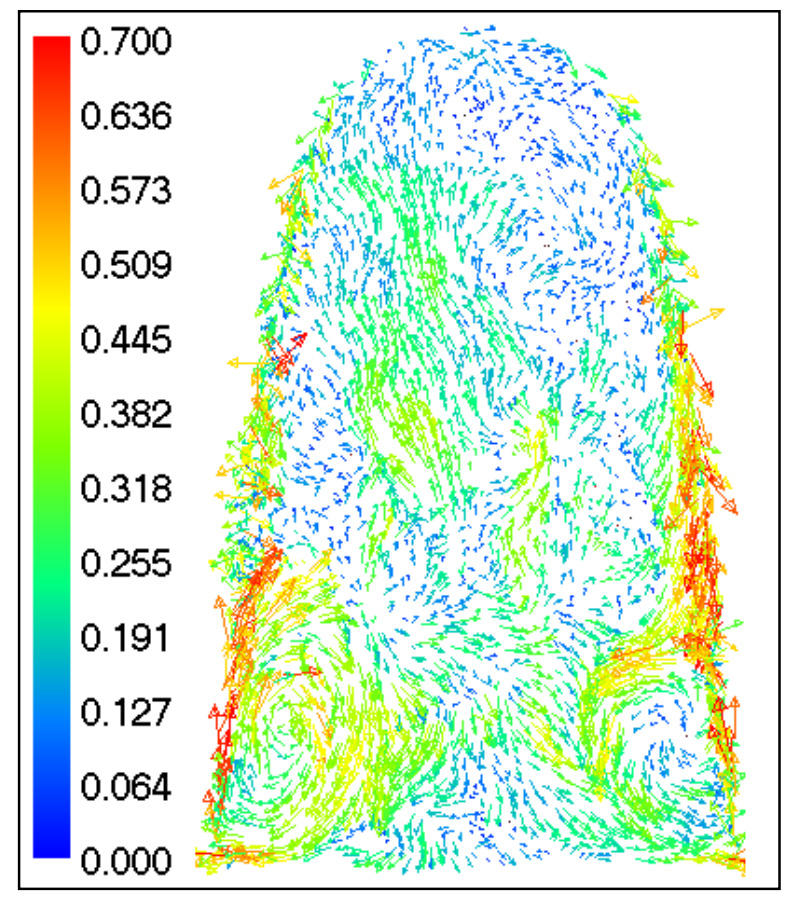

Figure 10. Transient flow pattern calculated with 3D LES $[\mathrm{m} / \mathrm{s}]$

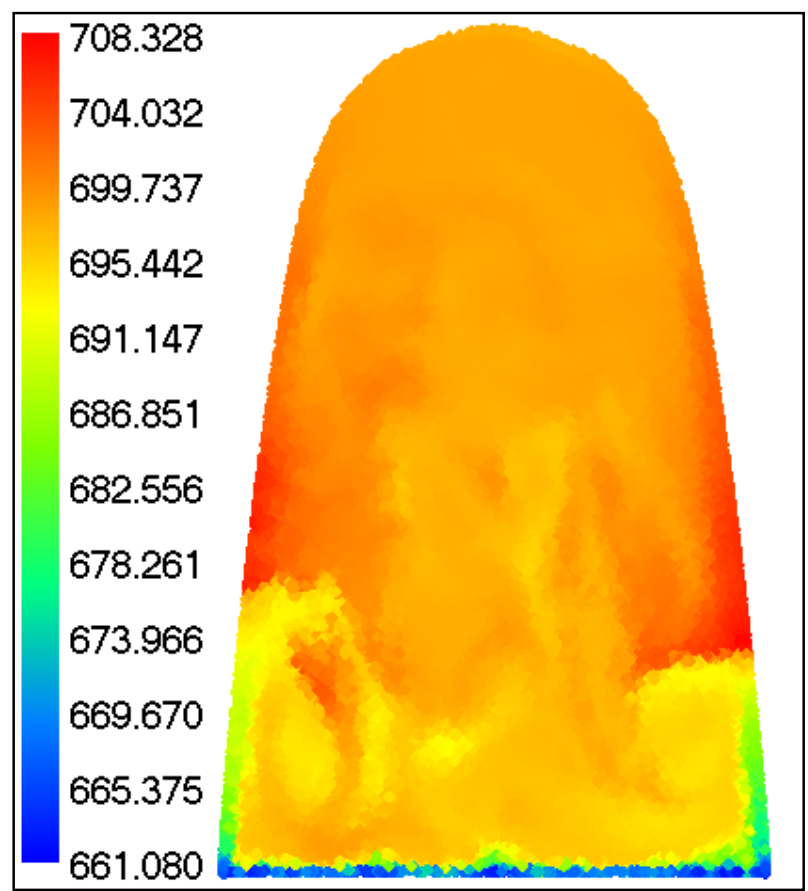

Figure 11. Transient temperature distribution calculated with $3 \mathrm{D}$ LES $\left[{ }^{\circ} \mathrm{C}\right]$

with cold crucible. Proc. of the Int. Seminar on Heating by Internal Sources. Padua (Italy), September 12-14, 2001, pp. 21-28

[3] Baake, E.; Nacke, B.; Umbrashko, A.; Jakovics, A.: LES Modelling of Heat and Mass Transfer in Turbulent Recirculated Flows. Proc. of the $5^{\text {th }}$ Int. PAMIR Conference, Ramatuelle, France, Sept. 16-20, 2002, Vol. I, p. 173-178

[4] Buchholz, A., Schmitz, W., Engler, S. Anwendung inductiver Magnetsonden bei Stroemungsmessung in fluessigem Aluminium. Metall 46 (1992) Nr. 10, S. 1018-1023

[5] Baake, E.: Grenzleistungs- und Aufkohlungsverhalten von Induktions-Tiegelöfen. Fortschrittberichte VDI, Reihe 19, Nr. 74, VDI-Verlag Düsseldorf, 1994

[6] Baake, E.; Nacke, B.; Umbrashko, A.; Jakovics, A.: Turbulent flow dynamics, heat transfer and mass exchange in the melt of induction furnaces. Proc. of the Int. Seminar on Heating by internal sources, Padova, 2001, pp. 47 - 53.

[7] Fluent 5 User's guide. Fluent Inc. 1999

[8] Smagorinsky J.S.: General circulation model of the atmosphere. Monthly Weather Review. p.91-99, 164, 1963.

[9] Lilly D.K.: On the application of the eddy viscosity concept in the inertial subrange of turbulence. NCAR Manuscript, 123, 1966. 